\title{
The solution to the cosmological constant problem
}

\author{
Brian Craig ${ }^{1}$ \\ ${ }^{1}$ McDonald Grove, West Lakes, Adelaide SA 5021, Australia
}

\begin{abstract}
To account for the very low mass density associated with Dark Energy and the Cosmological Constant, a new approach to the ground state of empty space is presented. The resulting model for the vacuum state associated with empty space proposes a crystalline-like texture for the chromatic structure of empty space. This vacuum state has the appropriate mass density and predicts acceleration for the Universe expansion. Furthermore, the model predicts that this texture is anisotropic and may lead to measurable changes in the production of electron and positron pairs by gamma rays incident on a solid crystal of low mass density such as graphite.
\end{abstract}

Keywords: dark energy theory

\section{Introduction}

Analysis of the cosmic microwave background (CMB) measurements by the NASA Wilson and the ESA Planck projects has supported the Standard Model of the Universe with a uniform and expanding universe [1]. While the CMB mesaurements are uniform to 1 part in 100,000, several anomolies in the CMB [2] have created substantial interest.

The expanding uniform density sphere model was used by Friedmann (1922) [3] to derive expressions for an expanding Universe. Friedmann's work provided the theoretical foundation for the Hubble equation, $\dot{a}=H a$, where $a(\mathrm{t})$ is the scaling factor and $\mathrm{H}$ is the Hubble constant. Freidmann's equation is

$$
H^{2}=\frac{8 \pi}{3} G \rho-\frac{k}{a^{2}}
$$

where $\mathrm{G}$ is the gravitational constant, $\rho$ is the energy density of the expanding sphere, and $\mathrm{k}$ is the index for the curviture of the universe. The particular density $\rho=\rho_{c}$ that gives a flat universe, which corresponds to $k=0$, is the critical energy density.

Dark energy is associated with a very low energy (mass) density for the Cosmological Constant $(\Lambda)$. However there has been unresolved disagreement, known as the Cosmological Constant Problem [4-6], between the theoretical estimations of a very high value the for mass density of the vacuum state, and the very low non-zero value derived from astonomical observations.

This paper presents a model for the vacuum state that includes the contributions from the electronic and quantum chromatic (colour charge) structure. The model describes the properties of the vacuum state, including the the observed low mass density. Here the term vacuum state means the ground state of apparently empty space. Furthermore a viable experiment is proposed that may confirm the proposed crystalline colour structure of the ground state. 
In section 2 the key concepts that explains the very low value for the mass density are introduced. In section 3 the model for the ground state is described. In section 4 a proposed experiment based on a variation the well known conversion of gamma rays to electron-positron pairs, to test the prediction by the model of anisotropic colourtexture.

\section{Mass Density for the Coloured Ground State of Massless Coloured Bosons}

The model, which will be described in Section 3, is built on the proposition that the vacuum state of empty space is composed of densely textured chromatic boson content. The model uses a crystal field of colour (and anti colour) charge in which other massless bosons transport electronic charge as Bloch states. These Bloch states have pionlike colour/anticolour charge, spin and electronic charge characteristics.

The distance between lattice colour/anticolour sites is the order of $10^{-15} \mathrm{~m}$, the characteristic distance for the strong nuclear force. Equal numbers of positively and negatively charged pions $\left(\pi^{+}\right.$and $\left.\pi^{-}\right)$experience the same cystal field and therefore produce exactly the same charge density.

\subsection{Estimation of the Energy Density}

New vacuum is created as the Universe expands. Two assumptions are the change in energy is zero for the increase in volume $\mathrm{d} \Omega$, and the vacuum is uniform The energy expression for all vacuum in the Universe, of volume $\Omega$, is

$$
0=\rho_{v} \Omega c^{2}+U_{e l}
$$

where $\mathrm{c}$ is the velocity of light, $\rho_{v}$ is the mass density of the vacuum state and $\mathrm{U}_{\mathrm{el}}$ is the net electrostatic energy of the pion-pion interactions. The universe is assumed to be finite size such as a Poincare Sphere (positive curviture) or a three-torus (flat universe) [7].

Although both positive and negative charge densities are precisely equal, a critical error is made if the electrostatic attraction between the unlike charges is assumed to be exactly equal in magnitude and opposite in sign to the electrostatic repulsion of like charges, to give $\mathrm{U}_{\mathrm{el}}=0$. The latter term (for like charges) contains the electrostatic repulsion of a pion with itself. This very small positive contribution should not be included. Therefore the contribution to $\mathrm{U}_{\mathrm{el}}$ from each pion should be a very small negative value. The result for $\mathrm{U}_{\mathrm{el}}$ is

$$
U_{e l}=-N_{\pi} U_{\pi} \Omega
$$

where $\mathrm{U}_{\pi}$, the value of one electrostatic pion self-replusion energy is and $\mathrm{N}_{\pi}$ is the number of pion-like bosons per cubic metre of vacuum. Therefore the expression for $\rho_{v}$ is

$$
\rho_{v}=U_{\pi} N_{\pi} c^{-2} .
$$

The electronic density for one charged pion Bloch State is $\mathrm{e} \Omega^{-1}$ where e is the electronic charge. By intergrating over the Universe, yields the following expression for the $\mathrm{U}_{\pi}$

which is solved to give

$$
U_{\pi}=\frac{e^{2}}{4 \pi \epsilon_{o} \Omega^{2}} \iint d^{3} \boldsymbol{r}_{1} d^{3} \boldsymbol{r}_{2} \frac{1}{\left|\boldsymbol{r}_{1}-\boldsymbol{r}_{\mathbf{2}}\right|}
$$

$$
U_{\pi}=6 / 5 \frac{e^{2}}{4 \pi \epsilon_{o} R}
$$

where $\mathrm{R}$ is the radius of the Universe. An estimate for $\mathrm{U}_{\pi}$ can be obtained from the current value $4.4 \times 10^{26}$ metres [8]. The resulting value for $\mathrm{U}_{\pi}$ is approximately $6.3 \times 10^{-55}$ Joules.

If the distance between charged pions in the lattice is assumed to be $10^{-15} \mathrm{~m}$, then there are $10^{45}$ pions per cubic metre.

The resulting energy density is therefore approximately $6.3 \times 10^{-10} \mathrm{Jm}^{-3}$. Dividing by the square of the velocity of light, yields a mass density of $\rho_{v}=6.9 \times 10^{-27} \mathrm{kgm}^{-3}$. This value is consistent with the critical mass density of $9.2 \mathrm{x}$ $10^{-27} \mathrm{kgm}^{-3}$ given by the Friedman equation for a flat universe $(\mathrm{k}=0)$ and estimates [8] for the fraction of mass for dark energy for the Universe (70\%) and $\mathrm{H}=70 \mathrm{kms}^{-1} \mathrm{Mpc}^{-1}$. The value for colour density of course depends on the inverse cube of the average inter-pion distance. 
This vacuum energy density decreases as $1 / R$, although not constant, is significantly slower than mass density which decreases as $1 / \mathrm{R}^{3}$. Rewriting the Friedmann's equation as

$$
\dot{a}^{2}=\frac{8 \pi G}{3} \rho a^{2}-k
$$

leads to the observation [6] that if $\rho$ decreased faster than $\mathrm{R}^{-2}$ then the right hand side would decrease and the Universe will slow, contrary to the observed acceleration. A rate of $\rho$ proportional to $1 / \mathrm{R}$ is consistent with an increasing right hand side and acceleration.

Of note is the minimal difference between two very large numbers gives rise to a vacuum energy. The number of charge pions within the vacuum is $\Omega$ multipled by $10^{45}$. The effect therefore is a result of a difference just one in $3.57 \times 10^{125}$. Particularly remarkable is that this term leads to the largest contribution to the total mass in the Universe.

\section{Model}

The ground state for empty space is required to be transparent to normal matter at energies below the particleantiparticle pair creation threshold, where such new particles have rest mass. There should not be any transfer of momentum or energy when such matter pass through vacuum.

\subsection{Pion-like Bloch state moving within the Crystal Field.}

Translational symmetry gives rise to delocalised and massless Bloch wave-functions [10] for the pion-like bosons, $\varphi_{n}$, which are described by the expression

$$
\varphi_{n}(\boldsymbol{k}, \boldsymbol{r})=(\Omega)^{-1 / 2} e^{i \boldsymbol{k} \cdot \boldsymbol{r}} u_{n}(\boldsymbol{r})
$$

where $\mathbf{r}$ is the position vector in real space, $\mathbf{k}$ is the crystal wavevector, $\mathrm{n}$ is the band index number, $\mathrm{u}_{\mathrm{n}}(\mathbf{r})$ has the periodicity of the lattice. These Bloch functions describe the electronic charge carriers within the lattice.

Each pion Bloch state has a component in each unit cell, called a Wannier Function [10]. This Bloch state is written as

$$
\varphi_{n}(\boldsymbol{k}, \boldsymbol{r})=\left(N_{U C}\right)^{-1 / 2} \sum_{i=1}^{i=N_{U C}} W_{n}\left(\boldsymbol{r}-\boldsymbol{R}_{\boldsymbol{i}}\right) e^{-i \boldsymbol{k} \cdot \boldsymbol{R}_{\boldsymbol{i}}}
$$

where $\mathrm{N}_{\mathrm{UC}}$ is the number of unit cells. The $i$ th unit cell has position $\mathbf{R}_{\mathbf{i}}$, and $\mathrm{W}_{\mathrm{n}}\left(\mathbf{r}-\mathbf{R}_{\mathbf{i}}\right)$ is the Wannier Function describing the spatial distribution of a pion at site $\mathbf{R}_{\mathrm{i}}$. The pion has a particular colour-anticolour combination charcateristic for the component quark and antiquark for that site $\mathbf{R}_{\mathbf{i}}$. While the combination cancels to colourless, this colour charge participates in the pion-gluon interaction, and is shown.

The pion-like bosons interact with a periodic array of sites where each site has gluon-like character. Here each gluon-like site contains one colour charge and one anticolour charge and total spin $S=1$. A step by step outline of this interaction is shown in Figure 1 for a simple one dimensional case.

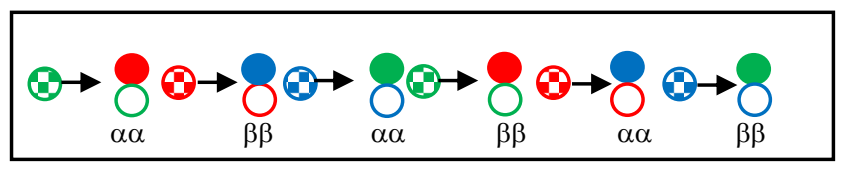

Figure 1: Progression of a continuous sequence of pions (checked circles) through a one-dimensional array of gluons. Each gluon contains one colour charge (solid circle) and one anticolour charge (empty circle).

Colour charges are shown as solid circles and anticolour charges by empty circles. The unit cell consists of six such lattice sites, each with a different colour/anticolour combination and spin. Spin is shown by the terms $\alpha$ and $\beta$, as used in Quantum Chemistry, for up and down spin respectively. The array is antiferromagnetic.

The Wannier-like states are shown as checked colour circles to denote composition of the colour/anticolour charge. These have no net colour. The change in colour of the pion indicates that an interaction with the crystal field has occurred. The ordered and coherent change in colour maintains the textured colour crystal field of the vacuum. 
The same process has occurred in the same position in all unit cells as expected for interacting extended states rather than localised particles.

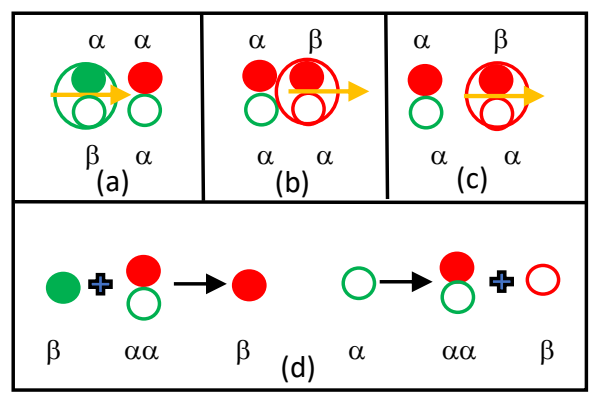

Figure 2: A pion is shown in (a) with one green colour and one antigreen colour charge, contained within the large circle, interacts with a gluon, containing one red and one antigreen colour charge. The pion colour absorbs the gluon, thereby changing the colour charge and emits a gluon, thereby charges the anticolour charge as shown in (d). The pion changes to red/antired colour combination (b) and the new gluon occupies the correct position (c). Also shown are notional spins for each colour.

On approaching a lattice site, the quark component of the pion absorbs a gluon of the characteristic colour/anticolour of that site. This gluon was just emitted by the pion ahead, and likewise the present pion antiquark component emits a similar gluon. This process is shown in Figure 2. The newly emitted gluon always reoccupies the position of the former gluon, and always possesses the same colour and spin. The pion has changed the colour of the quark and antiquark components when moving from $\mathbf{R}_{\mathrm{j}}$ to $\mathbf{R}_{\mathrm{j}+1}$, as shown in Figure 2(d) These colour changes occur with the same periodicity of the one dimenional lattice as expected for a Bloch state.

The pion, through the constituent quark and antiquark, interact with the lattice by continuously recycling gluons, always maintaining the exact colour/anticolour composition of the lattice. The gluons change the colour of the pions such that all pions always have the same colour/anticolour for each particular position (and Wannier Function component) this particle occupies.

The crystal field is maintained by the coherent interaction of gluons and pions and is self supporting. The gluons and pions otherwise would have a velocity of the speed of light as expected for massless bosons. The crystal field is the collective effect arising from the interaction between the two sets of bosons moving in opposite directions, like a standing colour wave. This colour crystal field contains the energy density.

\subsection{The Crystal Field of Six Colour Charge Types.}

The three dimensional crystal consists of a regular array of the three colours and three anticolours in the form of a lattice of gluon-like lattice sites. The distance between two neighbouring pions is assumed to be the order of $10^{-15}$ metres.

The unit cell of the crystal field is required to have no net spin or colour. This cell has two colour charges (one for spin $\alpha$, one with spin $\beta$ ) for each colour and anticolour. The crystal field therefore has six colour charges and six anticolour charges and therefore six gluon-like lattice sites.

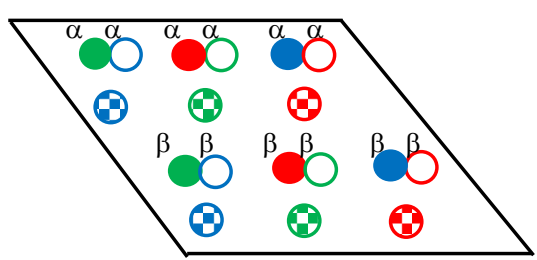

Figure 3: The unit cell of six gluons and six pions. A notional spin for the gluons is shown. 
The resulting unit cell is shown above in Figure 3. The colour charges of the crystal field are arranged sequentially in red, blue, then green, in each of the positive $\mathrm{x}, \mathrm{y}$ and $\mathrm{z}$ directions. While the gluons occupy sites within a cubic grid, the crystal colour is a triclinic system (though with three equal angles).

A larger portion of the crystal in shown in figure 4. The two solid yellow arrows represent the key translation vectors to neighbouring unit cells in the same layer. The dashed yellow arrow indicates the translation to the red/antigreen cell below (not shown) on the next layer.

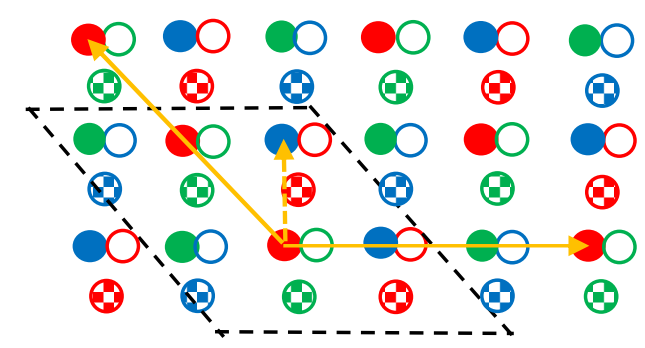

Figure 4: The three translation vectors defining the triclinic lattice. The dashed line connects the red colour charge in the displayed layer with a red colour charge in the layer below.

The unit cell was generated by the process

$$
0=3\left(\pi^{+}+\pi^{-}\right)+3 \mathrm{~g}(\alpha \alpha)+3 \mathrm{~g}(\beta \beta)
$$

where "g" is a massless gluon and $\alpha$ is spin up $(+1 / 2)$ and $\beta$ is spin down $(-1 / 2)$. The crystal field is antiferromagnetic. Each layer has rows of $\operatorname{spin} u p(\alpha, \alpha)$ and spin down $(\beta, \beta)$ gluons.

A complete crystal is generated by this process. Repeating the unit cell process $\left(\mathrm{N}^{3} / 6\right.$ times) generates a cubic prism of $\mathrm{NxNxN}$ cells, $\mathrm{N}^{3}$ massless gluons and mobile $\mathrm{N}^{3}$ massless pion-like electronic charge carriers.

The model so far has assumed a simple shape, like a cube, for the crystal in to provide an example to illustrate the concepts which is consistent with a three-torus [7]. As the universe expands the colour lattice is strained and cleaves. There is the immediate creation of new unit cells within the sudden gap and translational symmetry is maintained.

\subsection{Charge Density and Pion Flow.}

All pions within a column move together. The shape of the path is dictated by the topology of the finite Universe. A three-torus may give rise to continuous unidirectional paths reflecting the properties of this topology [7].

All colour charges in a path are required to move coherently along the same path and speed. A limit for the density of colour charges should exit. Otherwise fermion quark components of the pions of the same velocity, colour and spin will be too close to each other.

The key assumption in section 2 is the change in energy arising from additional vacuum formed from the expanding Universe is zero. This assumption leads to the very low value for the vacuum energy density. This assumption is reasonable as otherwise the total energy will continue to increase with the expanding Universe. This zero value is the sum of the negative $\mathrm{U}_{\mathrm{el}}$ and the positive energy stored in the crystal field. The first term is determined by the pion density, and the second by the related colour density.

The size of the Universe, $R$, increases as the Universe gets older and $U_{\pi}$ decreases as $1 / R$. In order to maintain the zero sum, the colour density may adjust slightly such that the energy stored per colour decreases. The strong nuclear interaction is short range and only a minor change in colour density may result.

\section{Investigating the Model.}

The preferred approach to investigate the proposed textured model for the vacuum is likely to be a scattering experiment using the particles associated the Standard Model of Particle Physics. 
Bragg diffraction, which is often a good choice for investigating crystals, requires an elastic collision where momentum is transferred between the diffracting parton and the crystal. The femtometre size of the crystal unit cell in the vacuum state leads to very high energy diffracting particles and substantial momentum changes.

The diffracting particle, with initial momentum, $\mathbf{p}_{\mathbf{i}}$, experience a change in momentum, to $\mathbf{p}_{\mathbf{i}} \mathrm{h} \mathbf{h}$, where $\mathrm{h}$ is the reduced Plank constant and $\mathbf{G}$ is a reciprical lattice vector, the order of $2 \pi / \mathrm{L}$ where $\mathrm{L}$ is the size of the crystal unit cell (near $10^{-15} \mathrm{~m}$ ). The collision being elastic requires the crystal momentum to gain by hG. This is far too high for a crystal of massless components and a very low energy density. Any diffracted beam could at best be extremely weak. Bragg Diffraction is currently assessed as a difficult choice for an initial experimental test of the model.

However an experiment based on electron-positron pair creation from gamma rays may probe the pion Bloch functions through the weak interaction. The electronic structure of the vacuum ground state is the massless charged pion structure. This electronic structure will display the cystalline and the antiferromagnetic-colour structure of the crystal field which are both non-spherical.

The absorption of a gamma ray by this ground state to produce, through the weak interaction, an electron from a negative pion-like Bloch function and a positron from a positive pion-like Bloch function (along with a neutrino and an antineutrino) is interesting.

The vacuum ground state does not have sufficient mass/crystal-momentum density to conserve momentum in this pair production processes. Instead a heavy atom is required where the gamma ray absorption occurs within the strong fields within this atom and the generated positron-electron pair propagate away in different directions. Momentum, with some energy, is conserved by transfer to the heavy atom.

Rather than a localised heavy atom, an interesting variation would be to have a crystal of light material. The momentum will not be transferred to a single spherically symmetric atom, but instead to the crystal through lattice vibrations.

The rate of pair generation may be partially determined by the co-alignment of this crystal with the vacuum colour lattice and pion structure. If the crystal of lightweight material is also anistropic then the anistropy of lattice vibrations (phonons) and pair generation may be particularly sensitive to the crystalline co-alignment. Good examples may be graphite phase of carbon and the similar phase of boron nitride (BN).

An experiment would first determine a suitable orientation of graphite or BN that yields near constant positronelectron pairs, and then see whether the pair production rate is influenced by the co-aligning the graphite-like phonon structure to the initially unknown orientation of the vacuum structure. The rotation of the earth will naturally change the relative vacuum alignment and potentially produce a measureable change in pair production rate.

\section{Conclusions}

A straightforward explanation for the origin and magnitude of Dark Energy has been presented. Dark Energy is the product of the finite size of the universe and the non-zero electronic charge density of the vacuum state. In turn this charge density is derived from the colour density arising from the Strong Interaction.

A crystal lattice model has been proposed for the colour and electronic charge content. However experimental testing of this crystalline textured vacuum would help confirm the assumptions that underpin this model.

\section{References}

[1] Bucher M 2015 Nuclear and Particle Physics Proceedings 267-269 245

[2] Bucher M 2015 International Journal of Modern Physics D 241530004

[3] Friedmann A 1922 Zeitschrift fur Physik A 10377

[4] Weinberg S 1989 Reviews of Modern Physics 611

[5] Banks T 2004 Physics Today 5746

[6] Unpublished comments of Carroll S https://ned.ipac.caltech.edu/level5/March01/Carroll/Carroll1.html

[7] Luminet J-P 2016 Universe 21

[8] Bars I, and Teming J 2009 Extra Dimensions in Space and Time, Springer, New York, NY, USA

[9] WMAP and Planck data for the Universe https://www.nasa.gov/mission_pages/planck/news/planck20130321.html 
[10] Kohn W 1959 Physical Review 115809 